\title{
TWISTED SURFACES WITH VANISHING CURVATURE IN GALILEAN 3-SPACE
}

\author{
MUSTAFA DEDE, CUMALI EKICI, WENDY GOEMANS, AND YASIN ÜNLÜTÜRK \\ Department of Mathematics, Kilis 7 Aralik University, 79000 Kilis, Turkey \\ E-mail address: mustafadede@kilis.edu.tr \\ Department of Mathematics-Computer, Eskişehir Osmangazi University, 26480 Eskişehir, \\ TURKEY \\ E-mail address: cekici@ogu.edu.tr \\ Faculty of Economics and Business, KU Leuven, Warmoesberg 26, 1000 Brussels, Belgium \\ E-mail address: wendy.goemans@kuleuven.be \\ Department of Mathematics, Kirklareli University, 39100 Kirklareli, Turkey \\ E-mail address: yasin_unluturk@yahoo.com
}

\begin{abstract}
In this work we define twisted surfaces in Galilean 3-space. In order to construct these surfaces, a planar curve is subjected to two simultaneous rotations, possibly with different rotation speeds. The existence of Euclidean rotations and isotropic rotations leads to three distinct types of twisted surfaces in Galilean 3-space. Then we classify twisted surfaces in Galilean 3-space with zero Gaussian curvature or zero mean curvature.
\end{abstract}

\section{INTRODUCTION}

A surface of revolution or a rotational surface is one of the first examples of a surface in basically every book on elementary differential geometry, see for instance [10]. Its simple construction, being the trace of a planar curve subjected to a rotation, is not only attractive from a geometrical point of view, but also makes a surface of revolution a shape that is found abundantly in real life. The catenoid, obtained by rotating a catenary, is one of the elementary minimal surfaces.

Extending this class of surfaces of revolution, is the class of helicoidal surfaces or generalized helicoids, see for example [10]. Indeed, helicoidal surfaces arise when a planar curve is rotated about an axis, while simultaneously it is translated in the direction of that rotation axis.

Another possible generalization of surfaces of revolution originates when the planar curve is not only rotated about an axis in the supporting plane, but simultaneously is also rotated in that supporting plane. This construction which subjects a planar curve to two simultaneous rotations is first mentioned in [10] where the resulting surface is called a twisted surface. It is used there as a generalization of the Möbius strip and the twisted Klein bottle.

2010 Mathematics Subject Classification. 53A10, 53A35, 53A40.

Key words and phrases. Galilean 3-space, twisted surface, flat surface, minimal surface. 
The third author, together with I. Van de Woestyne, defined twisted surfaces in Minkowski 3-space and classified those with constant Gaussian curvature or constant mean curvature, see [7, 8, 9]. Later, in [11], a second type of twisted surfaces in Minkowski 3-space is considered by other authors.

In this article, we define three types of twisted surfaces in Galilean 3-space. The study of the geometry of this non-Euclidean space was initiated thoroughly by Röschel in [14]. Lately, several authors used it as ambient space in their research, see e.g. $[1,4,5,6,12,16]$ for examples on special surfaces.

For the twisted surfaces in Galilean 3-space we will examine flatness (that is, zero Gaussian curvature) and minimality (zero mean curvature) conditions.

First we recall the necessary preliminaries from Galilean 3-space. Then we define three types of twisted surfaces in Galilean 3-space after which we classify the flat and the minimal ones.

\section{Preliminaries}

From [14] we recall here the necessary preliminaries from Galilean 3-space, for more details, see [1].

Pointing out an absolute figure $\{\omega, f, I\}$ in the 3 -dimensional real projective space, the Galilean 3-space $\mathbb{G}^{3}$ arises in a Cayley-Klein fashion. Here $\omega$ is the absolute plane, $f$ the absolute line and $I$ the fixed elliptic involution of points of $f$. Then homogeneous coordinates $\left(x_{0}: x_{1}: x_{2}: x_{3}\right)$ are introduced such that $\omega$ is given by $x_{0}=0, f$ by $x_{0}=x_{1}=0$ and $I$ by $\left(0: 0: x_{2}: x_{3}\right) \mapsto\left(0: 0: x_{3}:-x_{2}\right)$. With regard to the six-parameter group of motions of $\mathbb{G}^{3}$, apart from the absolute plane, there exist two classes of planes in $\mathbb{G}^{3}$ : Euclidean planes which contain $f$ and in which the induced metric is Euclidean and isotropic planes that do not contain $f$ and in which the induced metric is isotropic. Also, there are four types of lines in $\mathbb{G}^{3}$ : isotropic lines which intersect $f$, non-isotropic lines which do not intersect $f$, non-isotropic lines in $\omega$ and the absolute line $f$.

In affine coordinates defined by $\left(x_{0}: x_{1}: x_{2}: x_{3}\right)=\left(1: x_{1}: x_{2}: x_{3}\right)$, the distance between two points $P_{i}=\left(x_{i}, y_{i}, z_{i}\right)$ with $i \in\{1,2\}$ is defined by

$$
d\left(P_{1}, P_{2}\right)=\left\{\begin{array}{cl}
\left|x_{2}-x_{1}\right| & \text { if } x_{1} \neq x_{2}, \\
\sqrt{\left(y_{2}-y_{1}\right)^{2}+\left(z_{2}-z_{1}\right)^{2}} & \text { if } x_{1}=x_{2} .
\end{array}\right.
$$

In these coordinates a plane $a x+b y+c z+d=0$ is Euclidean if $a \neq 0$ and $b=c=0$ and isotropic otherwise. A vector $\vec{a}=(x, y, z)$ is isotropic if $x=0$ and non-isotropic otherwise. The Galilean scalar product of two vectors $\vec{a}=(x, y, z)$ and $\vec{b}=\left(x_{1}, y_{1}, z_{1}\right)$ is defined by

$$
\langle\vec{a}, \vec{b}\rangle=\left\{\begin{array}{cl}
x x_{1} & \text { if } x \neq 0 \text { or } x_{1} \neq 0 \\
y y_{1}+z z_{1} & \text { if } x=x_{1}=0
\end{array}\right.
$$

A vector $\vec{a}$ is a unit vector if $\|\vec{a}\|:=\sqrt{\langle\vec{a}, \vec{a}\rangle}=1$. The Galilean cross product of two vectors $\vec{a}=(x, y, z)$ and $\vec{b}=\left(x_{1}, y_{1}, z_{1}\right)$ (not both isotropic vectors) is defined as

$$
\vec{a} \wedge \vec{b}=\left|\begin{array}{ccc}
0 & e_{2} & e_{3} \\
x & y & z \\
x_{1} & y_{1} & z_{1}
\end{array}\right|
$$


For standard coordinates $(x, y, z)$, the $x$-axis is non-isotropic while the $y$-axis and the $z$-axis are isotropic. The $y z$-plane, $x=0$, is Euclidean and the $x y$-plane and the $x z$-plane are isotropic.

In order to define twisted surfaces, we need the two types of rotation in $\mathbb{G}^{3}$. A Euclidean rotation about the non-isotropic $x$-axis is given by

$$
\left(\begin{array}{l}
x^{\prime} \\
y^{\prime} \\
z^{\prime}
\end{array}\right)=\left(\begin{array}{ccc}
1 & 0 & 0 \\
0 & \cos \theta & \sin \theta \\
0 & -\sin \theta & \cos \theta
\end{array}\right)\left(\begin{array}{l}
x \\
y \\
z
\end{array}\right)
$$

where $\theta$ is the Euclidean angle. An isotropic rotation is given by

$$
\left(\begin{array}{l}
x^{\prime} \\
y^{\prime} \\
z^{\prime}
\end{array}\right)=\left(\begin{array}{lll}
1 & 0 & 0 \\
\theta & 1 & 0 \\
0 & 0 & 1
\end{array}\right)\left(\begin{array}{l}
x \\
y \\
z
\end{array}\right)+\left(\begin{array}{c}
c \theta \\
c \\
\frac{2}{2} \\
0
\end{array}\right)
$$

where $\theta$ is the isotropic angle and $c \in \mathbb{R}_{0}$. Here the bundle of invariant planes is given by $z=$ constant. Under this isotropic rotation a point $P_{1}=\left(x_{1}, y_{1}, z_{1}\right)$ of $\mathbb{G}^{3}$ is moved over an isotropic circle of radius $c$ in the isotropic plane parameterized by $z=z_{1}$. An isotropic circle is a (Euclidean) parabola in an isotropic plane.

Finally, we define curvatures of a surface in $\mathbb{G}^{3}$. For a surface in $\mathbb{G}^{3}$ parameterized by

$$
\varphi\left(v^{1}, v^{2}\right)=\left(x\left(v^{1}, v^{2}\right), y\left(v^{1}, v^{2}\right), z\left(v^{1}, v^{2}\right)\right),
$$

denote the first order derivatives for $i \in\{1,2\}$ by $\varphi_{, i}=\frac{\partial \varphi}{\partial v^{i}}\left(v^{1}, v^{2}\right)$. Here we always assume that the surfaces are admissible, that is, its tangent plane is nowhere a Euclidean plane. The unit normal vector $N$ of the surface is defined by

$$
N=\frac{\varphi_{, 1} \wedge \varphi_{, 2}}{w} \quad \text { where } w=\left\|\varphi_{, 1} \wedge \varphi_{, 2}\right\| .
$$

The coefficients of the second fundamental form are given by

$$
L_{i j}=\left\langle\frac{\varphi_{, i j} x_{, 1}-x_{, i j} \varphi_{, 1}}{x_{, 1}}, N\right\rangle=\left\langle\frac{\varphi_{, i j} x_{, 2}-x_{, i j} \varphi_{, 2}}{x_{, 2}}, N\right\rangle .
$$

The Gaussian curvature $K$ and the mean curvature $H$ of the surface are defined by

$$
K=\frac{L_{11} L_{22}-L_{12}^{2}}{w^{2}} \quad \text { and } \quad 2 H=\sum_{i, j=1}^{2} g^{i j} L_{i j}
$$

where

$$
g^{1}=\frac{x_{, 2}}{w}, g^{2}=-\frac{x_{, 1}}{w} \text { and } g^{i j}=g^{i} g^{j} \text { for } i, j \in\{1,2\}
$$

\section{Twisted surfaces in Galilean 3-SPaCe}

A twisted surface in $\mathbb{G}^{3}$ is a surface that is constructed similar as in Euclidean or Minkowski 3-space.

Definition 3.1. A twisted surface in $\mathbb{G}^{3}$ is a surface that is traced out by a planar curve, the profile curve, on which two different simultaneous rotations are performed, which can have different rotation speeds and of which one keeps invariant the supporting plane of the profile curve. 
Because of the existence of the different kinds of planes and rotations in $\mathbb{G}^{3}$, different types of twisted surfaces are to be defined. The supporting plane of the profile curve can indeed be a Euclidean plane, which only contains isotropic vectors, or an isotropic plane, which contains both isotropic and non-isotropic vectors. For the rotations there is either one or there are two possibilities. These choices lead to three possible types of twisted surfaces in $\mathbb{G}^{3}$ in the following way.

3.1. Type $\mathbf{I}$ twisted surface in $\mathbb{G}^{3}$. We start with a profile curve in a Euclidean plane and rotate it about a Euclidean axis perpendicular to that supporting plane. Without losing generality, we assume that the profile curve $\alpha$ lies in the $y z$-plane and is parameterized by $\alpha(t)=(0, f(t), g(t))$ with $f$ and $g$ real functions. A Euclidean rotation about an axis parallel to the non-isotropic $x$-axis is performed on the profile curve,

$$
\begin{aligned}
\left(\begin{array}{l}
0 \\
a \\
0
\end{array}\right)+\left(\begin{array}{ccc}
1 & 0 & 0 \\
0 & \cos (b s) & \sin (b s) \\
0 & -\sin (b s) & \cos (b s)
\end{array}\right) & \left(\begin{array}{c}
0 \\
f(t) \\
g(t)
\end{array}\right) \\
& =\left(\begin{array}{c}
0 \\
a+f(t) \cos (b s)+g(t) \sin (b s) \\
-f(t) \sin (b s)+g(t) \cos (b s)
\end{array}\right)
\end{aligned}
$$

with $a, b \in \mathbb{R}$. Simultaneously, the profile curve is rotated using an isotropic rotation for which the bundle of invariant planes is given by $z=$ constant, thus,

$$
\left(\begin{array}{ccc}
1 & 0 & 0 \\
s & 1 & 0 \\
0 & 0 & 1
\end{array}\right)\left(\begin{array}{c}
0 \\
a+f(t) \cos (b s)+g(t) \sin (b s) \\
-f(t) \sin (b s)+g(t) \cos (b s)
\end{array}\right)+\left(\begin{array}{c}
c s \\
c s^{2} \\
2 \\
0
\end{array}\right)
$$

with $c \in \mathbb{R}_{0}$. Therefore, up to a transformation, a type I twisted surface in $\mathbb{G}^{3}$ is parameterized by

$$
\varphi(s, t)=\left(c s, f(t) \cos (b s)+g(t) \sin (b s)+\frac{c}{2} s^{2},-f(t) \sin (b s)+g(t) \cos (b s)\right) .
$$

Remark 3.1. The rotation of the profile curve in its supporting Euclidean plane cannot be an isotropic rotation since there exists no isotropic rotation that keeps invariant a Euclidean plane. Therefore, starting with a profile curve in a Euclidean plane only one type of twisted surfaces can be generated.

3.2. Type II twisted surface in $\mathbb{G}^{3}$. Now we start with a profile curve $\alpha$ in an isotropic plane which we can assume to be, without losing generality, $\alpha(t)=$ $(f(t), g(t), 0)$ with $f$ and $g$ real functions. On this profile curve two simultaneous isotropic rotations are performed, one for which the bundle of invariant planes is given by $z=$ constant, that is,

$$
\left(\begin{array}{l}
a \\
0 \\
0
\end{array}\right)+\left(\begin{array}{ccc}
1 & 0 & 0 \\
b s & 1 & 0 \\
0 & 0 & 1
\end{array}\right)\left(\begin{array}{c}
f(t) \\
g(t) \\
0
\end{array}\right)+\left(\begin{array}{c}
b c s \\
\frac{b^{2} c}{2} s^{2} \\
0
\end{array}\right)
$$


where $a, b, c \in \mathbb{R}$ with $c \neq 0$ and simultaneously one for which the bundle of invariant planes is given by $y=$ constant, thus,

$$
\left(\begin{array}{lll}
1 & 0 & 0 \\
0 & 1 & 0 \\
s & 0 & 1
\end{array}\right)\left(\begin{array}{c}
a+f(t)+b c s \\
b s f(t)+g(t)+\frac{b^{2} c}{2} s^{2} \\
0
\end{array}\right)+\left(\begin{array}{c}
d s \\
0 \\
\frac{d}{2} s^{2}
\end{array}\right)
$$

with $d \in \mathbb{R}_{0}$. So, up to a transformation, a type II twisted surface in $\mathbb{G}^{3}$ is parameterized by

$$
\varphi(s, t)=\left(f(t)+(b c+d) s, b s f(t)+g(t)+\frac{b^{2} c}{2} s^{2},(a+f(t)) s+\left(b c+\frac{d}{2}\right) s^{2}\right) .
$$

3.3. Type III twisted surface in $\mathbb{G}^{3}$. Finally, again we start with a profile curve $\alpha=(f(t), g(t), 0)$, with $f$ and $g$ real functions, that lies in the isotropic $x y$-plane. Now we perform on $\alpha$ an isotropic rotation for which the bundle of invariant planes is given by $z=$ constant,

$$
\left(\begin{array}{l}
0 \\
a \\
0
\end{array}\right)+\left(\begin{array}{ccc}
1 & 0 & 0 \\
b s & 1 & 0 \\
0 & 0 & 1
\end{array}\right)\left(\begin{array}{c}
f(t) \\
g(t) \\
0
\end{array}\right)+\left(\begin{array}{c}
b c s \\
\frac{b^{2} c}{2} s^{2} \\
0
\end{array}\right)
$$

with $a, b, c \in \mathbb{R}$ and $c \neq 0$. This time, the simultaneous rotation is a Euclidean one about the non-isotropic $x$-axis,

$$
\left(\begin{array}{ccc}
1 & 0 & 0 \\
0 & \cos s & \sin s \\
0 & -\sin s & \cos s
\end{array}\right)\left(\begin{array}{c}
f(t)+b c s \\
a+b s f(t)+g(t)+\frac{b^{2} c}{2} s^{2} \\
0
\end{array}\right) .
$$

Hence, up to a transformation, a type III twisted surface in $\mathbb{G}^{3}$ is parameterized by

$$
\begin{aligned}
\varphi(s, t)=(f(t)+b c s, \cos s(a+b s f(t) & \left.+g(t)+\frac{b^{2} c}{2} s^{2}\right), \\
& \left.-\sin s\left(a+b s f(t)+g(t)+\frac{b^{2} c}{2} s^{2}\right)\right) .
\end{aligned}
$$

For all three types of twisted surfaces the parameter $b$ allows the two simultaneous rotations to have different rotation speeds. If $b=0$ the parameterizations of the twisted surfaces reduce to that of surfaces of revolution in $\mathbb{G}^{3}$ which were defined and studied in [6].

\section{Flat and minimal twisted SuRfaces in $\mathbb{G}^{3}$}

We examine for the three types of twisted surfaces in $\mathbb{G}^{3}$ when they have vanishing Gaussian curvature, i.e., when they are flat and when they have vanishing mean curvature, i.e., when they are minimal. Here we copy the terminology that is used for surfaces in Euclidean 3-space.

Concerning minimal surface in $\mathbb{G}^{3}$, we mention the following important theorem from [14] which characterizes all minimal surfaces in $\mathbb{G}^{3}$.

Theorem 4.1 ([14]). The minimal surfaces in Galilean 3-space are cones whose vertex lies on the absolute line $f$ and ruled surfaces of type $C$, that is, conoidal surfaces having the absolute line $f$ as the directional line at infinity. 
A type $\mathrm{C}$ ruled surface is a ruled surface parameterized by

$$
\varphi(s, t)=(s, f(s), 0)+t\left(0, \beta_{2}(s), \beta_{3}(s)\right)
$$

with $f, \beta_{2}$ and $\beta_{3}$ at least three times continuous differentiable real functions such that $\beta_{2}(s)^{2}+\beta_{3}(s)^{2}=1$ for all $s$.

In this section we perform patch computations on the parameterizations that are presented in the previous section. Hereby we always assume that the constant $b$ appearing in those parameterizations is non-zero. A study of the case $b=0$, that is, surfaces of revolution in $\mathbb{G}^{3}$ with vanishing curvature, can be found in [6].

4.1. Zero curvature type I twisted surfaces in $\mathbb{G}^{3}$. For a type I twisted surface in $\mathbb{G}^{3}$ parametrized by

$$
\varphi(s, t)=\left(c s, f(t) \cos (b s)+g(t) \sin (b s)+\frac{c}{2} s^{2},-f(t) \sin (b s)+g(t) \cos (b s)\right)
$$

with $b, c \in \mathbb{R}_{0}$ we calculate the Gaussian curvature and the mean curvature to be

$$
K=\frac{\left(f^{\prime} g^{\prime \prime}-f^{\prime \prime} g^{\prime}\right)\left[b^{2}\left(f g^{\prime}-f^{\prime} g\right)+c\left(f^{\prime} \sin (b s)-g^{\prime} \cos (b s)\right)\right]-b^{2}\left(f^{\prime 2}+g^{\prime 2}\right)^{2}}{c^{2}\left(f^{\prime 2}+g^{\prime 2}\right)^{2}}
$$

and

$$
H=\operatorname{sgn}(c) \frac{f^{\prime} g^{\prime \prime}-f^{\prime \prime} g^{\prime}}{2\left(f^{\prime 2}+g^{\prime 2}\right)^{3 / 2}}
$$

Here and in the remainder of the paper we often drop the parameter of the functions $f$ and $g$ for reasons of readability. Primes denote derivation and by $\operatorname{sgn}$ we mean the sign function. Remark that $f^{\prime}$ and $g^{\prime}$ can not be both identically zero since then the surface is not admissible.

Considering the flatness condition, we prove the following non-existence result.

Theorem 4.2. Excluding the surfaces of revolution, there do not exist flat type I twisted surfaces in $\mathbb{G}^{3}$.

Proof. If the expression $K=0$ is derived with respect to $s$ one obtains

$$
b c\left(f^{\prime} \cos (b s)+g^{\prime} \sin (b s)\right)\left(f^{\prime} g^{\prime \prime}-f^{\prime \prime} g^{\prime}\right)=0 .
$$

Now we know that $b$ and $c$ are non-zero. If $f^{\prime} \cos (b s)+g^{\prime} \sin (b s)=0$ then, because of the linear independency of the sine and cosine functions, $f^{\prime}=g^{\prime}=0$. But then the surface is not admissible so we exclude this case. Thus $f^{\prime} g^{\prime \prime}=f^{\prime \prime} g^{\prime}$. If this is inserted in the expression $K=0$ it reduces to $b^{2}\left(f^{\prime 2}+g^{\prime 2}\right)^{2}=0$, which leads to a contradiction. Therefore, when we exclude the surfaces of revolution, there do not exist flat type I twisted surfaces in $\mathbb{G}^{3}$.

With concern to the minimality condition, we prove the following theorem.

Theorem 4.3. Excluding the surfaces of revolution, a type I twisted surface in $\mathbb{G}^{3}$ is minimal if and only if it is a type $C$ ruled surface parameterized by

$$
\begin{aligned}
\varphi(s, t)=\left(c s, q \cos (b s)+\frac{c}{2} s^{2},\right. & -q \sin (b s)) \\
& +t(0, p \cos (b s)+\sin (b s), \cos (b s)-p \sin (b s)),
\end{aligned}
$$

here $p, q \in \mathbb{R}$. 


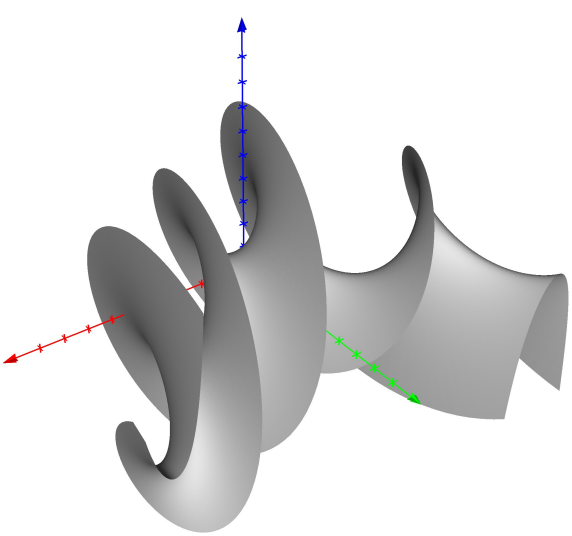

(a) A minimal type I twisted surface which is a type $\mathrm{C}$ ruled surface, parameterized by (1) with $b=3, c=4, p=q=1$.

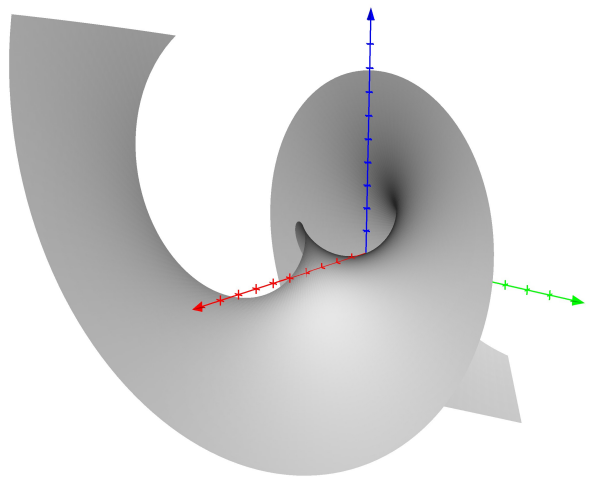

(b) A minimal type III twisted surface which is a type $\mathrm{C}$ ruled surface, parameterized by (6) with $a=2, b=\frac{1}{2}, c=4, p=1$.

Figure 1. Minimal type I and type III twisted surfaces.

Proof. There are three cases in which $H=0$. It can be that $f^{\prime}=0$ and $g^{\prime} \neq 0$ or vice versa. Then we obtain, up to a transformation, parameterization (1) with $p=0$ or (1) with the sine and cosine functions interchanged, respectively. If $f^{\prime} \neq 0$ and $g^{\prime} \neq 0$ then $H=0$ if and only if $\frac{f^{\prime \prime}}{f^{\prime}}=\frac{g^{\prime \prime}}{g^{\prime}}$, which leads to $g(t)=p f(t)+q$ with $p, q \in \mathbb{R}$ and $p \neq 0$. Therefore, if necessary after reparameterizing with $f(u)=t$, we obtain parameterization (1).

The following corollary is immediate from Theorem 4.3 and Theorem 2 in [6].

Corollary 4.1. A type I twisted surface in $\mathbb{G}^{3}$ is a minimal surface if and only if its profile curve is an isotropic straight line.

See Figure 1(a) for a drawing of a minimal type I twisted surface.

Remark that for type I twisted surfaces the flatness and minimality condition are not equivalent, contrary to the equivalency of these conditions for type I surfaces of revolution, see [6].

4.2. Zero curvature type II twisted surfaces in $\mathbb{G}^{3}$. For a type II twisted surface in $\mathbb{G}^{3}$ parametrized by

$$
\varphi(s, t)=\left(f(t)+(b c+d) s, b s f(t)+g(t)+\frac{b^{2} c}{2} s^{2},(a+f(t)) s+\left(b c+\frac{d}{2}\right) s^{2}\right)
$$

with $a, b, c, d \in \mathbb{R}$ and $b c d \neq 0$, the expression for the Gaussian curvature is

$$
\begin{aligned}
K=\frac{1}{w^{4}}\left[\left\{b f^{\prime}\left(a b c+(b c+d)^{2} s\right)-b(b c\right.\right. & +d) f f^{\prime} \\
\left.+(2 b c+d)(b c+d) g^{\prime}\right\} & (a+b c s+f)\left(f^{\prime} g^{\prime \prime}-f^{\prime \prime} g^{\prime}\right) \\
& \left.\quad-f^{\prime 2}\left\{b(a+(b c+d) s) f^{\prime}+(b c+d) g^{\prime}\right\}\right]
\end{aligned}
$$


and the mean curvature is

$$
\begin{aligned}
H=\frac{1}{2 w^{3}}\left[-b f^{\prime 3}(a(b c\right. & \left.+2 d)+(b c+d)^{2} s\right)-b(b c+d) f f^{\prime 3} \\
& \left.-d(b c+d) f^{\prime 2} g^{\prime}+(b c+d)^{2}(a+b c s+f)\left(f^{\prime} g^{\prime \prime}-f^{\prime \prime} g^{\prime}\right)\right] .
\end{aligned}
$$

Here $w^{2}=f^{\prime 2}(a+b c s+f)^{2}+\left(b(d s-f) f^{\prime}+(b c+d) g^{\prime}\right)^{2}$. Again, remark that $f^{\prime}$ and $g^{\prime}$ can not be both identically zero since then the surface is not admissible.

The following classification theorem is valid.

Theorem 4.4. When excluding the surfaces of revolution, a type II twisted surface in Galilean 3-space is flat/minimal if and only if it is (up to a transformation) either

(1) an isotropic plane, parameterized by

$$
\varphi(s, t)=\left(f(t), b s f(t)+g(t)+\frac{b^{2} c}{2} s^{2}, s f(t)+\frac{b c}{2} s^{2}\right)
$$

(2) a type $C$ ruled surface (parabolic cylinder) parameterized by

$$
\varphi(s, t)=\left(p+(b c+d) s, b p s+\frac{b^{2} c}{2} s^{2},(a+p) s+\left(b c+\frac{d}{2}\right) s^{2}\right)+t(0,1,0),
$$

(3) a cyclic surface (parabolic sphere) parameterized by (2) with g satisfying,

$$
g(t)=\frac{(b c+d) f^{2}(t)+2 a d f(t)-p}{2 c(b c+d)},
$$

where $a, b, c, d, p \in \mathbb{R}$ and $b c d \neq 0$.

Proof. We give here the proof for the flat surfaces, for the minimal surfaces one has to proceed similarly. The expression $K=0$ is a second degree polynomial in $s$, hence all the coefficients must be zero. The coefficient of the quadratic term is $-b^{2}(b c+d)^{2} f^{\prime}\left(f^{\prime 3}+c\left(f^{\prime \prime} g^{\prime}-f^{\prime} g^{\prime \prime}\right)\right)$. This leads to three cases to consider.

Case $1 d=-b c$.

The coefficient of the linear term of the expression $K=0$ reduces to

$$
a b^{3} c^{2} f^{\prime}\left(f^{\prime} g^{\prime \prime}-f^{\prime \prime} g^{\prime}\right)
$$

If $a$ is zero, then $K=0$ and we obtain parameterization (3). For $f^{\prime}=0$ the surface is not admissible and $f^{\prime} g^{\prime \prime}=f^{\prime \prime} g^{\prime}$ leads to a contradiction or to the previous subcase since then $a b f^{\prime}=0$ from the constant term in the condition $K=0$.

Case $2 f^{\prime}=0$.

In this case we obtain parameterization (4).

Case $3 f^{\prime 3}=c\left(f^{\prime} g^{\prime \prime}-f^{\prime \prime} g^{\prime}\right)$.

Here we have to assume that $g^{\prime} \neq 0$ since otherwise also $f^{\prime}=0$ and in that case the surface is not admissible. Thus we can solve the condition for an expression for $f^{\prime \prime}$ which is then inserted in the equation $K=0$. The linear term of that equation then has as a coefficient

$$
\frac{b d}{c} f^{\prime 3}\left((b c+d) f f^{\prime}-(b c+d) c g^{\prime}+a d f^{\prime}\right) .
$$

Since $b d f^{\prime} \neq 0$, the last term of this coefficient must be zero. Integrating that term leads to condition (5). 
It is easy to see that in parameterization (2), when condition (5) is valid, the components satisfy,

$$
((b c+d) x+a d)^{2}-2 c(b c+d)^{2} y-2 d(b c+d)^{2} z=2 p(b c+d)+a^{2} d^{2}
$$

which characterizes a cyclic surface, see [15]. Moreover, setting $u=f(t)+(b c+d) s$ and $v=(a+f(t)) s+\left(b c+\frac{d}{2}\right) s^{2}$, parameterization (2), incorporating condition (5), is rewritten to

$$
\varphi(u, v)=\left(u, \frac{1}{2 c}\left(u+\frac{a d}{b c+d}\right)^{2}+\frac{p}{2 c(b c+d)}-\frac{a^{2} d^{2}}{2 c(b c+d)^{2}}, 0\right)+v\left(0,-\frac{d}{c}, 1\right)
$$

from which it is immediate that it is the parameterization of a type $\mathrm{C}$ ruled surface. Remark that although it is not immediate seen from the expressions for $K$ and $H$, it is clear from Theorem 4.4 that for type II twisted surfaces the minimality and the flatness conditions are equivalent. This is also the case for type II surfaces of revolution, see [6].

4.3. Zero curvature type III twisted surfaces in $\mathbb{G}^{3}$. A type III twisted surface in $\mathbb{G}^{3}$ parameterized by

$$
\varphi(s, t)=(f(t)+b c s, h(s, t) \cos s,-h(s, t) \sin s)
$$

with $h(s, t)=a+b s f(t)+g(t)+\frac{b^{2} c}{2} s^{2}$ where $a, b, c \in \mathbb{R}$ and $b c \neq 0$ has

$$
\begin{aligned}
K=\frac{1}{w^{4}}\left[h\left(f^{\prime \prime} g^{\prime}-f^{\prime} g^{\prime \prime}\right)\left(h f^{\prime}\left(h-h_{s s}\right)-2 h_{s}\left(b c h_{t}-f^{\prime} h_{s}\right)\right)\right. & \\
& \left.-\left(h_{t}\left(f^{\prime} h_{s}-b c h_{t}\right)-f^{\prime} h h_{s t}\right)^{2}\right]
\end{aligned}
$$

and

$$
\begin{aligned}
H=\frac{1}{2 w^{3}}\left[f^{\prime 2}\left(h f^{\prime}\left(h-h_{s s}\right)-2 h_{s}\left(b c h_{t}-f^{\prime} h_{s}\right)\right)\right. & \\
& \left.-2 b c f^{\prime}\left(h_{t}\left(f^{\prime} h_{s}-b c h_{t}\right)-f^{\prime} h h_{s t}\right)+b^{2} c^{2} h\left(f^{\prime \prime} g^{\prime}-f^{\prime} g^{\prime \prime}\right)\right]
\end{aligned}
$$

with $w^{2}=\left(b c h_{t}-f^{\prime} h_{s}\right)^{2}+h^{2} f^{\prime 2}$. Subscripts denote derivation with respect to that variable or those variables. Also for these type III twisted surfaces $f^{\prime}$ and $g^{\prime}$ can not be zero simultaneously since then the surface is not admissible. Analougous as for the type I twisted surfaces, also a non-existence result for flat type III twisted surfaces in $\mathbb{G}^{3}$ is valid.

Theorem 4.5. When excluding the surfaces of revolution, there exists no flat type III twisted surface in Galilean 3-space.

Proof. The condition that $K$ is zero is a fourth degree polynomial in $s$ with the fourth order coefficient equal to $-16 b^{6} c^{2} f^{\prime 4}$. All the coefficients of that polynomial must be zero. That means that $f^{\prime}=0$. When this is inserted in the condition $K=0$, it reduces to $-16 b^{2} c^{2} g^{\prime 4}=0$. But then this leads to a contradiction since $f^{\prime}$ and $g^{\prime}$ can not be both zero since then the surface is not admissible.

Similar one proofs the following classification theorem of minimal type III twisted surfaces in $\mathbb{G}^{3}$. 
Theorem 4.6. A minimal type III twisted surface in $\mathbb{G}^{3}$ is a type $C$ ruled surface parameterized by

$$
\begin{aligned}
\varphi(s, t)=(p+b c s, \cos s(a & \left.+p b s+\frac{b^{2} c}{2} s^{2}\right) \\
& \left.-\sin s\left(a+p b s+\frac{b^{2} c}{2} s^{2}\right)\right)+t(0, \cos s,-\sin s)
\end{aligned}
$$

with $p \in \mathbb{R}$.

This minimal type III twisted surface has constant Gaussian curvature. See Figure 1(b) for a drawing of a minimal type III twisted surface.

Remark that in Theorem 4.6 it is not necessary to exclude the type III surfaces of revolution since there do not exist minimal ones, see [6].

\section{Conclusion, Further Research AND ACKNOWledgment}

We defined three types of twisted surfaces in Galilean 3-space and studied flatness and minimality conditions for these surfaces. We proved that there do not exist flat type I nor flat type III twisted surfaces in $\mathbb{G}^{3}$. On the other hand all the flat type II twisted surfaces in $\mathbb{G}^{3}$ are also minimal and vice versa.

There also exists a pseudo-Galilean 3 -space $\mathbb{G}_{1}^{3}$ which is related to Galilean 3space in a similar way as Minkowski 3-space is related to Euclidean 3-space, see for instance $[2,3,13]$. Without going into detail here, the pseudo-Galilean scalar product of two vectors $\vec{a}=(x, y, z)$ and $\vec{b}=\left(x_{1}, y_{1}, z_{1}\right)$ in $\mathbb{G}_{1}^{3}$ is defined by

$$
\langle\vec{a}, \vec{b}\rangle_{1}=\left\{\begin{array}{cl}
x x_{1} & \text { if } x \neq 0 \text { or } x_{1} \neq 0, \\
y y_{1}-z z_{1} & \text { if } x=x_{1}=0
\end{array}\right.
$$

Because of this there exist four types of isotropic vectors $\vec{a}=(0, y, z)$ in $\mathbb{G}_{1}^{3}$ : spacelike ones (if $y^{2}-z^{2}>0$ ), timelike ones (if $y^{2}-z^{2}<0$ ) and two types of lightlike ones (if $y= \pm z$ ). Analougously as in Minkowski 3-space, instead of the trigonometric functions, the hyperbolic functions must be used to describe rotations. Therefore it is clear that one can also define different types of twisted surfaces in $\mathbb{G}_{1}^{3}$. This could be a subject for further research. Also, the constant curvature twisted surfaces in $\mathbb{G}^{3}$ could be examined in future works, although the computations might be significant harder in some cases.

For the calculations in this article we used the computer algebra system Maple. The drawings in this article are made with VisuMath, www.visumath.be.

We are very grateful to the referee for the valuable comments which improved the first version of this manuscript.

\section{REFERENCES}

[1] M. E. Aydin and A. O. Ögrenmiş, Spherical product surfaces in the Galilean space, Konuralp Journal of Mathematics, 4 (2016), 290-298.

[2] M. E. Aydin, A. O. Ögrenmiş and M. Ergut, Classification of factorable surfaces in the pseudo-Galilean space, Glasnik Matematicki, 50 (2015), 441-451.

[3] M. Dede, Tube surfaces in pseudo-Galilean space, International Journal of Geometric Methods in Modern Physics, 13(5) (2016) :1650056.

[4] M. Dede, Tubular surfaces in Galilean space, Math. Commun., 18 (2013), 209-217.

[5] M. Dede, C. Ekici and A. Ceylan Çöken, On the parallel surfaces in Galilean space, Hacettepe Journal of Mathematics and Statistics 42(6) (2013), 605-615. 
[6] M. Dede, C. Ekici and W. Goemans, Surfaces of revolution with vanishing curvature in Galilean 3-space, accepted for publication in Journal of Mathematical Physics, Analysis, Geometry.

[7] W. Goemans and I. Van de Woestyne, Constant curvature twisted surfaces in 3-dimensional Euclidean and Minkowski space, Proceedings of the Conference RIGA 2014 Riemannian Geometry and Applications to Engineering and Economics Bucharest, Romania (2014), 117130.

[8] W. Goemans and I. Van de Woestyne, Twisted surfaces in Euclidean and Minkowski 3-space, Pure and Applied Differential Geometry: 2013, J. Van der Veken, I. Van de Woestyne, L. Verstraelen and L. Vrancken (Editors), Shaker Verlag (Aachen, Germany), 143-151.

[9] W. Goemans and I. Van de Woestyne, Twisted surfaces with null rotation axis in Minkowski 3-space, Results Math. 70(1) (2016), 81-93. DOI: 10.1007/s00025-015-0462-2.

[10] A. Gray, E. Abbena and S. Salamon (eds.) Modern Differential Geometry of Curves and Surfaces with Mathematica, Chapman \& Hall/CRC, Boca Raton, 2006.

[11] M. Grbović, E. Nešović and A. Pantić, On the second kind twisted surfaces in Minkowski 3-space, International Electronic Journal of Geometry 8(2) (2015), 9-20.

[12] Ž. Milin Šipuš and B. Divjak, Some special surfaces in the pseudo-Galilean space, Acta Math. Hungar., 118 (2008), 209-226.

[13] Ž. Milin Šipuš and B. Divjak, Surfaces of constant curvature in the pseudo-Galilean space, Internat. J. Math. Math. Sci., vol. 2012, Article ID 375264, 28 pages, 2012. DOI:10.1155/2012/375264

[14] O. Röschel, Die Geometrie des Galileischen Raumes, Bericht der Mathematisch-Statistischen Sektion in der Forschungsgesellschaft Joanneum, Bericht Nr. 256, Habilitationsschrift, Leoben, 1984.

[15] I. M. Yaglom, A simple non-Euclidean geometry and its physical basis, Springer-Verlag New York Inc, 1979.

[16] Z. K. Yüzbaşı and M. Bektaş, On the construction of a surface family with common geodesic in Galilean space $\mathbb{G}^{3}$, Open Phys., 14 (2016), 360-363.

Department of Mathematics, Kilis 7 Aralik University, 79000 Kilis, Turkey

E-mail address: mustafadede@kilis.edu.tr

Department of Mathematics-Computer, Eskişehir OsmangaZi University, 26480 Eskişehir, TURKEY

E-mail address: cekici@ogu.edu.tr

Faculty of Economics and Business, KU Leuven, Warmoesberg 26, 1000 Brussels, Belgium

E-mail address: wendy.goemans@kuleuven.be

Department of Mathematics, Kirklareli University, 39100 Kirklareli, Turkey

E-mail address: yasin_unluturk@yahoo.com 